Embodied Semantic Effects in Visual Word Recognition

Louise Connell

\&

Dermot Lynott

Lancaster University

Pre-print of:

Connell, L., \& Lynott, D. (2015). Embodied semantic effects in visual word recognition. In Y. Coello, \& M. Fischer (Eds.), Foundations of Embodied Cognition (Vol. 2): Conceptual and Interactive Embodiment. Hove, UK: Psychology Press.

Author Note

Louise Connell and Dermot Lynott, Department of Psychology, Lancaster University. The order of authorship is arbitrary.

Correspondence concerning this article should be addressed to Louise Connell or Dermot Lynott, Department of Psychology, Fylde College, Lancaster University, Bailrigg, Lancaster LA1 4YF, UK. Email: 1.connell@lancaster.ac.uk or d.lynott@lancaster.ac.uk 


\section{Embodied Semantic Effects in Visual Word Recognition}

\section{Introduction}

Words have meanings; on that much, psycholinguists are generally agreed. However, the issue of what "meaning" is, and why a word's semantic content affects how easily it is recognised, are matters of less consensus. Studies of visual word recognition typically ask participants to perform one of two key tasks: deciding whether a letter string is a valid word (lexical decision), or reading a word aloud (word naming). On the face of it, one should not have to access meaning in order to perform either of these tasks: knowing that "fabric" is a real word but "fabnic" is not, or being able to pronounce it correctly, does not have an obvious semantic requirement. Nonetheless, the meaning of a word affects how quickly it can be processed. "Fabric", for example, is recognised more quickly, and with fewer errors, than "factor", even when other word-level variables such as length, frequency, and so on, have been controlled.

Semantic effects are believed to happen in both tasks for similar reasons: active semantic content facilitates processing orthographic and/or phonological representations of the word (Coltheart, Rastle, Perry, Langdon, \& Ziegler, 2001; Harm \& Seidenberg, 2004; Hino \& Lupker, 1996; Plaut et al., 1996). That is, when a written word is presented, its orthographic representation spreads activation to semantic content. A word that achieves a high level of semantic activation will feed activation back to the orthographic and/or phonological representation, and facilitate a relatively rapid task response ("yes" in lexical decision; correct pronunciation in word naming). A word with a low level of semantic activation, however, will feed much less activation back to the orthographic and/or phonological representations, and facilitate the task response by only a small amount (if at all). Such semantic facilitation may not occur if the orthographic or phonological representations independently reach a sufficiently high level of activation to enable a task response. Very high frequency words, for example, may be 
recognised too quickly for semantic information to have a noticeable effect: as a general rule, low-frequency words such as "cad" benefit more from semantic facilitation than do highfrequency words like “cat” (e.g., Cortese \& Schock, 2013; de Groot, 1989; James, 1975). Nonetheless, the meaning of a word can play a role in early word recognition processes by mediating orthographic and/or phonological activation. ${ }^{1}$

Decades of research have shown that reading a word is easier if its semantics conform to certain characteristics. Broadly speaking, these effects can be divided into three distinct levels of remove:

- Level 1 pertains to the specific qualities of the semantic content of the word; that is, the nature of the information that comprises the referent concept. The more the referent of a concept is concrete (e.g., de Groot, 1989; James, 1975), perceptible (Connell \& Lynott, 2012a, 2014; Cortese \& Schock, 2013; Juhasz, Yap, Dicke, Taylor, \& Gullick, 2011; Yap, Pexman, Wellsby, Hargreaves, \& Huff, 2012), or affords interaction (Siakaluk, Pexman, Aguilera, Owen, \& Sears, 2008; Yap et al., 2012), then the easier its label will be to process. In general, it is easier to recognise words whose meanings are based on some sort of physical existence.

- Level 2 pertains to enumeration of the semantic content of the word; that is, the number of discrete aspects that can be listed for the referent concept. Having an ambiguous

${ }^{1}$ There are many disagreements between models of visual word recognition as to whether orthographic representations are lexicalised (Coltheart et al., 2001) versus distributed (Plaut et al., 1996; Seidenberg \& McClelland, 1989), or whether the entire removal of the semantic system would inevitably impair all word processing to some extent (Rogers, Lambon Ralph, Hodges, \& Patterson, 2004) versus leave lexical decision and word naming intact (Blazely, Coltheart, \& Casey, 2005). There is consensus, however, on the basic process just outlined. 
referent concept with many potential versions (Borowsky \& Masson, 1996; Yap et al., 2011; cf. Rodd et al., 2002), or many conceptual features (Pexman et al., 2002; Grondin et al., 2009), makes a word easier to process. Level 2 effects are one step removed from Level 1 because it is irrelevant whether or not the word's meaning has a physical basis. The actual semantic content of the features or meanings does not matter, and it is instead their sheer quantity or variety that appears to facilitate word recognition.

- Level 3 pertains to the company the word (and therefore its referent concept) keeps; what is sometimes called its semantic neighbourhood. That is, if a word tends to reappear with a large set of other words across contexts (Buchanan et al., 2001; Pexman et al., 2008; cf. Mirman \& Magnuson, 2008), or cues a large number of associates in free-association tasks (Buchanan et al., 2001; Duñabeitia, Avilés, \& Carreiras, 2008; Pexman et al., 2002), then it will be recognised more easily. By shifting the semantic focus away from the word's referent concept and towards its connections with other words, Level 3 effects are one step removed from Level 2. Here, the actual semantic content of the word (i.e., the information that comprises the referent concept) does not matter, but rather the quantity or variety of related words.

Embodied semantic effects constrain semantics to experience-based perceptual and motor information. Grounded theories of cognition hold that the conceptual system has effectively coopted perceptual, motor, affective, and other systems for the purpose of representation (Barsalou, 1999; Connell \& Lynott, 2014b; Coventry, Lynott, Cangelosi, Monrouxe, Joyce \& Richardson; Meteyard, Rodriguez Cuadrado, Bahrami, \& Vigliocco, 2012; Pulvermüller, 2005; Solomon \& Barsalou, 2004). The neural activation produced in these systems during perceptual, motor, etc. experience can be later partially re-enacted (or simulated) in order to represent information in conceptual processing. Such simulations are not restricted to mere re-enactment, however, but 
can be dynamically adapted and extended to predict aspects of entities and events that have not been directly experienced (e.g., Barsalou, 2009; Gallese, 2009). The net result is that the meaning of a word is processed using some of the same neural structures as those involved in perceptual, motor, etc. experience of its referent. Because embodied semantic effects focus on the specific nature of semantic content, they are Level 1 effects. Level 2 and 3 semantic effects, while they can be compatible with a grounded view of cognition, do not manifest it. For example, features of a tomato such as red and edible could be represented as an embodied visualgustatory-motor simulation or as an amodal network of symbols: they can be enumerated as $n=2$, regardless. Similarly, the word "tomato" may cue the words "red" and "fruit" regardless of the format of the referent concepts. Because their greater abstraction makes Level 2 and 3 effects agnostic to the underlying nature of the semantic content, they do not qualify as embodied effects.

In this chapter, we focus our attention on the range of embodied semantic effects that have been found in visual word recognition. Many different embodied effects have been elicited by distinct measures of sensorimotor information, and are associated with different theoretical accounts of why semantic content affects how quickly a word is recognised. Imageability effects emerge from considering semantic content as an imagistic representation, the theoretical tenet of Paivio's $(1986,2007)$ dual coding theory, which provides a grounding mechanism not unlike simulation-based accounts of meaning. ${ }^{2}$ Body-object interaction (Siakuluk et al., 2008;

${ }^{2}$ Although "concreteness effects" are a textbook finding in visual word recognition, many ostensible effects in the literature are in fact elicited from imageability ratings (e.g., Binder et al., 2005; Fliessbach et al., 2006; Sabsevitz et al., 2005), and are far from robust, disappearing in the presence of perceptual strength effects (Connell \& Lynott, 2012). Concreteness effects, therefore, are subsumed by other, more specific measures of the physical basis of semantic 
Tillotson, Siakaluk \& Pexman, 2008), relative embodiment (Sidhu, Kwan, Pexman \& Sakaluk, 2008), and sensory experience effects (Juhasz et al., 2011; Juhasz \& Yap, 2012) explicitly support the idea of semantic content as a sensorimotor simulation. Modality-specific perceptual strength effects (Connell \& Lynott, 2012a, 2014a, in prep) take this idea further, by considering semantic content as a sensorimotor simulation modulated by perceptual attention. Finally, we discuss the impact of embodied semantic effects on current models of visual word recognition.

\section{Types of Embodied Semantic Effects}

\section{Semantic Content as Imagistic Representation}

In Paivio’s (1986, 2007; see also Sadoski, McTigue, \& Paivio, 2012) dual coding theory, concepts comprise two types of representation: a verbal code that corresponds approximately (but not exactly) to word labels, and an imagistic code of modality-specific sensorimotor information that can give rise to conscious imagery when activated. These verbal and imagistic codes are linked, but not in a one-to-one mapping. While all words evoke a verbal code, only some connect directly to a corresponding imagistic code. Hence, words like "church" are processed relatively easily because they possess these direct connections to an imagistic representation, while words like "religion" take more effort because they can only connect indirectly via other verbal codes (e.g., verbal code of religion links to verbal code of church, which then accesses an imagistic representation). The directness of connection from word to image is effectively a gradation of grounding: some words are more easily represented in terms of sensorimotor mental imagery than others. An easily-imaged word will produce greater semantic activation (or at least produce it sooner) than a difficult-to-image word, and feed back activation to orthographic/phonological representations and facilitate word recognition.

Imageability ratings were designed to tap into this construct by measuring the ease of

content. 
generating a mental image for a particular word (Paivio, Yuille \& Madigan, 1968), and cover a Likert scale from 1 (arouse images with the greatest difficulty or not at all) to 7 (arouse images most readily). Low-imageability words, for example (Clark \& Paivio, 2004), include "however" $(\mathrm{M}=1.34)$ and "reason" $(\mathrm{M}=1.78)$, whereas high-imageability words include "cat" $(\mathrm{M}=6.80)$ and "sunset" $(\mathrm{M}=6.83)$. Imageability effects are perhaps the most widely-reported semantic effect in visual word recognition (e.g., Balota et al., 2004; Cortese \& Schock, 2013; de Groot, 1989; Juhasz et al., 2011; Strain, Patterson, \& Seidenberg, 1995; Yap et al., 2012): highimageability words are faster and more accurate to process because they can achieve a higher level of semantic activation and facilitate orthographic and/or phonological activation better than low-imageability words. Indeed, imageability is often the established semantic variable against which candidate semantic variables are measured (e.g., Connell \& Lynott, 2012; Juhasz, et al., 2011; Siakaluk et al., 2008). Although many demonstrations of imageability effects suggested that they were potentially confounded by other variables such as age of acquisition (Brown \& Watson, 1987; Cortese \& Khanna, 2007; Monaghan \& Ellis, 2002) and/or restricted to words with atypical spelling-sound mappings (Strain et al., 1995; Woollams, 2005), current evidence from large-scale regression studies has shown that imageability has a robust effect on visual word recognition above and beyond a large range of other sublexical, lexical, and semantic variables (e.g., Cortese \& Schock, 2013; Yap et al., 2012). Nonetheless, several studies have noted that imageability effects are stronger in lexical decision than in naming (e.g., Balota et al., 2004; Yap, et al., 2012). Balota and colleagues (2004; Chumbley and Balota, 1984; Cortese \& Balota, 2013) proposed that this task difference emerges because the nature of the lexical decision task leads semantic information to be prioritised: since non-words are always semantically devoid, whereas valid words are semantically laden, the word/nonword decision can be recast as a meaningful/nonmeaningful decision. Word naming, on the other hand, 
requires both words and nonwords to be pronounced, and so focusing on semantics does not offer the same strategic advantage.

However, imageability is not without its problems. Although it was intended to reflect the ease of generating mental imagery across a range of sensory modalities, imageability is instead heavily biased towards vision (Connell \& Lynott, 2012). By comparing imageability ratings with ratings of perceptual strength across five separate modalities - vision, sound, touch, taste, and smell - Connell and Lynott found that only vision had a consistent relationship with imageability. Other modalities were neglected (e.g., touch experience was unrelated to imageability) or misinterpreted (e.g., taste experience was negatively related to imageability). Connell and Lynott concluded that the visual bias in imageability had two probable causes: use of the word "image" in the rating instructions (which typically means a visual depiction), and people's disproportionately greater experience with visual imagery (which led all other modalities to appear difficult). Thus, imageability effects in visual word recognition are likely to represent only a small part of the potential semantic effects that have a perceptual basis.

\section{Semantic Content as Sensorimotor Simulation}

Upon reading a word, grounded theories of representation hold that semantic information is activated in the form of sensorimotor simulations that reflect the experience a person has had of the referent concept (e.g., Barsalou, 1999; Connell \& Lynott; 2014a; Meteyard et al., 2012). Recent semantic effects based on sensorimotor experience - body object interaction, relative embodiment and sensory experience ratings - explicitly link semantic activation with simulation (Juhasz et al., 2011; Juhasz \& Yap, 2012; Siakaluk et al., 2008). That is, when a word is being processed, semantic content is activated in the form of a sensorimotor simulation, which then feeds back activation to orthographic/phonological representations. Hence, words that have a 
high degree of perceptual or motor information in their simulation will have greater semantic activation and facilitate recognition of the word.

Body-object interaction ratings are an action-based measure, designed to capture the ease with which a person can physically interact with an object in the world. Individual objects (i.e., nouns) are rated on a 1-7 Likert scale, where a rating of 7 indicates that the human body can easily physically interact with a particular object, while a rating of 1 indicates that the human body cannot easily interact with it (Tillotson, Siakaluk, \& Pexman, 2008). For example, words with high body-object interaction scores include "toothbrush" $(\mathrm{M}=6.22)$ and "bicycle" $(\mathrm{M}=$ 6.33), while words with low body-object interaction scores include "lightning" $(\mathrm{M}=1.15)$ and "volcano" $(\mathrm{M}=1.33)$. Following the sensorimotor simulation view, higher body-object interaction ratings should facilitate visual word recognition. Indeed, Siakaluk et al. (2008) found that words with high body-object interaction scores were identified more quickly than those with low scores in lexical decision. Moreover, this effect of body-object interaction appeared independent of imageability (Siakaluk et al., 2008; Tillotson et al., 2008). In regression analyses over 512 words, Yap et al., (2012) provided further support for the role of body-object interaction in visual word recognition, finding it to be a significant predictor of response latencies in three different tasks (lexical decision task, go-no go lexical decision task, word naming). The effect was again independent of imageability, as well as a number of other lexical and sublexical variables including age of acquisition.

However, body-object interaction effects do not always appear when expected. In two large-scale regressions of nearly 1200 words apiece, Juhasz et al. (2011) found body-object interaction ratings had no effect on lexical decision performance once imageability and age of acquisition had been controlled. Furthermore, Wellsby and Pexman (2014) investigated whether the body-object interaction effect in word naming extended to children's visual word recognition. 
Due to higher error rates by children in this task, a composite performance measure combining response time and error rates measure was created. While older children (8-9 years) showed similar facilitation to adult participants, younger children (6-7 years) did not show any bodyobject interaction effect. Some studies using alternative visual word recognition paradigms have also failed to elicit body-object interaction effects (Hargreaves and Pexman, 2014; Yap et al., 2012). For example, Yap and colleagues employed a progressive demasking task in which a word was presented repeatedly, but rapidly alternated with a mask (e.g., \#\#\#\#). The duration of the mask was gradually reduced, while the duration of the word increased, until the participant indicated they had successfully identified the word. Yap et al. had hoped that that the progressive demasking task would offer greater sensitivity to semantic effects, citing several methodological advantages over lexical decision and naming (e.g., no need to create non-word distractor items, not influenced by articulatory factors). Nonetheless, body-object interaction did not facilitate performance. Thus, evidence is mixed for the facilitatory role of body-object interaction measure in visual word recognition.

The relative embodiment measure proposed by Sidhu et al., (2014) is another actionbased measure that is closely related to body-object interaction. Where body-object interaction ratings refer only to nouns, relative embodiment ratings refer only to verbs. Nonetheless, the operationalisation of both measures is very similar. For relative embodiment ratings, participants were asked to judge the degree to which the meaning of each verb involves the human body on a 1-7 Likert scale. Instructions directed participants to give higher ratings to any verb that is related to an action, state, or relation that easily involves the human body, and lower ratings to those that do not easily involve the human body. Verbs that were rated as high in relative embodiment include "jog" $(\mathrm{M}=6.53)$ and "scream" $(\mathrm{M}=6.36)$, while "forecast" $(\mathrm{M}=1.97)$ and "broaden" $(\mathrm{M}=2.23)$ were rated low in relative embodiment. Using regression analysis of 
lexical decision times for 392 verbs, Sidhu et al. found that relative embodiment was a significant predictor of response times, while imageability was not: words that were higher in relative embodiment were processed more quickly than those lower in relative embodiment, paralleling the trend observed for body-object interaction. The influence of relative embodiment was in addition to a set of lexical and sublexical control variables, including age of acquisition. Although only one study, Sidhu et al.'s relative embodiment measure can be combined with body-object interaction as demonstrations of action-based embodied semantic effects.

Sensory experience ratings also draw on the framework of sensorimotor simulation, but differ from body-object interaction and relative embodiment measures in focusing on perception rather than action. Sensory experience ratings (Juhasz, et al., 2011; Juhasz \& Yap, 2012) refer to the degree of sensory experience evoked by a word; that is, the actual sensation experienced upon reading the word. Participants rate words on a 1-7 Likert scale, with 1 indicating the word evokes no sensory experience, and 7 indicating the word evokes a strong sensory experience. Examples of words with a high sensory experience rating include "garlic" $(\mathrm{M}=6.56)$ and "cozy" (5.90), while "least" $(\mathrm{M}=1.10)$ and "could" $(\mathrm{M}=1.17)$ are words with a low sensory experience rating. Juhasz et al. (2011) used a large-scale regression analysis to test for the role of sensory experience in lexical decision of over 2200 monosyllabic words, using data from the E-Lexicon and British Lexicon projects (Balota et al., 2007; Keuleers, Lacey, Rastle, \& Brysbaert, 2012). They found that sensory experience ratings were a significant predictor of both response times and accuracy, with higher ratings leading to faster response times and greater accuracy. Importantly, the effect of sensory experience was over and above a number of sublexical and lexical variables (including age of acquisition), as well as above imageability. These effects were further replicated in Juhasz and Yap's (2013) analysis of 4,738 mono and disyllabic words, with reliable sensory experience effects in both lexical decision and word naming latencies (though 
effects were weaker in the latter task). For lexical decision, at least, sensory experience effects also replicate in French (Bonin, Méot, Ferrand, \& Bugaïska, in press), although analysis of 1659 words found that sensory experience ratings did not predict word naming latencies. Finally, one analysis has examined sensory experience ratings alongside body-object interaction (i.e., perceptual and motor experience together): in regression of lexical decision times for 1200 words, Juhasz et al. (2011) found that sensory experience ratings were a reliable predictor over and above body-object interaction ratings, although body-object interaction did not itself have an effect. Overall, then, findings suggest that words with greater sensory experience provide a processing advantage in visual word recognition, though more so in lexical decision than in word naming.

In summary, while there is evidence of a role for body-object interaction, relative embodiment, and sensory experience ratings in visual word recognition, there remains some open empirical and methodological questions. First is the issue of word class. For body-object interaction and relative embodiment, it is not clear at this point whether bodily/action experience applies to all classes of words, as only nouns and verbs have been normed so far (with different, but related, instructions). Whether adjectives and adverbs can be easily normed with current instructions has yet to be resolved (e.g., "quickly" might or might not relate to bodily action: it depends on the verb it modifies). In a similar vein, Juhasz and Yap (2012) mention that sensory experience ratings acted as a significant predictor of lexical decision and naming times only for nouns, and not for verbs and adjectives. Hence, more work is needed to establish whether these sensorimotor semantic effects, given their physical basis, apply differently to different word classes.

Second is the apparent fragility of action-based body-object interaction effects. While there is evidence for the role of body-object interaction (and the similar measure of relative 
embodiment) in visual word recognition tasks such as lexical decision and naming, these effects sometimes disappear (e.g., Hargreaves \& Pexman, 2014; Juhasz et al., 2011; Yap et al., 2012). One possible reason is a confound with age of acquisition. It is difficult to draw conclusions about confounds from factorial experiments, given the loss of statistical power and potential for experimenter bias in selecting subsets of items, and the difficulties of adequately controlling extraneous variables (Balota et al., 2012). ${ }^{3}$ Happily, many recent studies use large-scale regression analyses over hundreds of words, which offer greater reliability in establishing effects. Tillotson et al. (2008) and Yap et al. (2012) both report significant effects of body-object interaction in their regression analyses of lexical decision performance, but neither include age of acquisition in their models. Juhasz et al. (2011) do include age of acquisition as a predictor of lexical decision, and body-object interaction no longer has an effect. Although Juhasz and colleages do not report the correlation between body-object interaction and age of acquisition, it is plausible that many of the first words acquired by children refer to physical objects that afford interaction. It should be noted that both body-object interaction and relative embodiment measures have also been tested in non-reading tasks, such as picture naming and syntactic classification (Sidhu et al., 2014), semantic categorisation (Tousignant \& Pexman, 2012), and imageability judgements (Wellsby et al., 2011). Such tasks require deeper processing than visual word recognition, and even require explicit assessment of meaning, and are thereby more likely to rely on semantic information than visual word recognition tasks. Thus, evidence for the role of body-object interaction and relative embodiment in such tasks should not automatically be assumed to provide evidence for a role in visual word recognition. It is therefore unclear how

${ }^{3}$ As an illustration of these difficulties, we refer to the well-known debate regarding imageability and age of acquisition in word naming (Ellis \& Monaghan, 2002; Monaghan \& Ellis, 2002; Strain et al., 1995; Strain, Patterson, \& Seidenberg, 2002). 
robust are action-based embodied semantic effects in visual word recognition, and to what extent they emerge independent of age of acquisition.

Finally, perception-based embodied semantic effects, in the form of sensory experience ratings, need to be separated more cleanly from other semantic variables. Most importantly, the precise relationship between sensory experience and imageability needs to be teased apart. Juhasz et al (2011) demonstrate that adding sensory experience to a regression model that already includes imageability still produces a significant effect on lexical decision performance. That is, sensory experience ratings explain some variance in word recognition that imageability does not. However, it is not clear whether the inverse may also hold: does adding imageability to a model that already contains SER account for a significant proportion of variance? In other words, could sensory experience ratings subsume imageability? Such analysis has yet to be conducted. Moreover, while body-object interaction and sensory experience ratings are similar in many respects, particularly in their complementary framing of semantic content as sensorimotor simulation, the precise relationship between measures has not been fully identified. To what extent do these action- and perception-based measured exert independent effects in visual word recognition? Are the effects of comparable sizes? Do they cancel each other out? Which is more important to word recognition, active bodily interaction with a concept or potentially passive perception of a concept? Only one study to date has examined both variables simultaneously (Juhasz et al., 2011), but failed to find an effect of body-object interaction (possibly because of the presence of age of acquisition, as outlined above). Quite simply, more work needs to be done to disentangle the roles of perceptual versus motor simulation in visual word recognition.

\section{Semantic Content as Sensorimotor Simulation Modulated by Perceptual Attention}

A recent extension to the "semantic content as sensorimotor simulation" approach has 
combined the notion of modality-specific representations with the proposal that the activation of sensory information may be modulated by perceptual attention (Connell \& Lynott, 2012b, 2014a, 2014b). The impact of sensorimotor simulation co-opting the perceptual system is that conceptual and perceptual processing share representational and attentional resources. We know from work in perception that selectively attending to a particular perceptual modality increases activation in the corresponding sensory cortex (Foxe, Simpson, Ahlfors, \& Saron, 2005; Langner et al., 2011), which in turn facilitates processing of stimuli in that modality (Spence, Nicholls, \& Driver, 2001; Töllner, Gramann, Müller, \& Eimer, 2009). The same attentional effects emerge in simulation of perceptual information; that is, directing attention toward a particular perceptual modality facilitates conceptual processing of information in that modality (Connell \& Lynott, 2012b; Connell, Lynott, \& Dreyer, 2012; van Dantzig et al., 2008). Since reading itself implicitly engages perceptual attention, Connell and Lynott (2012a, 2014a) proposed that the resulting preactivation of modality-specific perceptual systems was one of the main reasons for facilitation in the comprehension of language that refers to strongly-perceptual concepts. In this attentional modulation of meaning activation (AMMA) account, semantic effects in visual word recognition depend on the concurrent perceptual demands of the participant. The perceptual attention implicitly involved in reading pre-activates modality-specific cortices, which leads to faster simulation of modality-specific information (i.e., faster semantic activation), which in turn feeds back activation to orthographic/phonological representations and facilitates word recognition.

Lynott and Connell (2009, 2013) collected modality-specific perceptual strength ratings for a large number of concepts, where participants separately rated the extent to which they experience each concept by seeing, hearing, smelling, tasting or feeling through touch. Ratings ranged from 0 (not experienced at all through this sense) to 5 (greatly experienced through this 
sense). In this way, each concept is represented by a 5-value vector, rather than a single composite value as utilised by the measures earlier discussed (imageability, body-object interaction, relative embodiment, sensory experience). The peak perceptual strength of a word can be identified by the maximum rating in its dominant modality. Examples of words of high perceptual strength include "yellow" (visual $M=4.95$ ), "minty" (gustatory $M=4.95$ ), and “bleeping” (auditory $\mathrm{M}=4.95$ ), while "heaven" (auditory $\mathrm{M}=1.76$ ) and "atom" (visual $\mathrm{M}=$ 1.38) were rated as being of low perceptual strength.

Connell and Lynott (2012a) investigated the extent to which perceptual strength ratings could account for performance in visual word recognition tasks. In regression analyses over 592 words, they found that maximum perceptual strength (i.e., strength in the dominant modality) was a good predictor of both lexical decision and word naming performance, independent of various lexical and sublexical variables: responses to strongly perceptual words were faster and more accurate than for weakly perceptual words. This perceptual strength effect was above and beyond that of imageability, and, critically, imageability had no effect once perceptual strength had already been taken into account. Thus, perceptual strength subsumes imageability, which suggests that it is the extent of perceptual experience in a referent concept, rather than the ease of consciously generating perceptual imagery, that produces semantic effects in visual word recognition. More recently, Connell and Lynott (in prep) directly contrasted maximum perceptual strength and sensory experience ratings in accounting for lexical decision performance. While both measures were significant predictors of response times and accuracy, perceptual strength outperformed sensory experience. It appears that sensory experience ratings, which require participants to aggregate sensory experience across modalities, may lead to the loss of some valuable information that is retained when people rate the perceptual strength each modality separately. Thus, following the AMMA theory, perceptual strength can better capture 
the semantic facilitation that is brought about by aspects of semantic content being simulated more rapidly due to modality-specific perceptual attention during reading.

Connell and Lynott (2014a) further developed the contribution of the perceptual strength measure by identifying task-specific and modality-specific effects predicted by AMMA. For example, given that a lexical decision task requires people to identify word forms, it implicitly directs visual attention to meet this goal. In a word naming task, visual attention is similarly required to identify the word forms, but because the task requires that a word is correctly pronounced, attention is also implicitly directed to the auditory modality to plan and monitor speech output. Connell and Lynott suggested that the differential perceptual attention implicitly involved in these tasks would lead to differential facilitation of words according to the visual and auditory strength in their referent concepts. For example, AMMA theory predicts that words with high visual strength (e.g., cloud) should be recognised more quickly than words with low visual strength (e.g., salty) in both lexical decision and word naming tasks. However, due to the additional engagement of auditory attention in the naming task, words that have a high auditory strength (e.g., noisy) should also receive a facilitatory boost relative to words with low auditory strength (e.g., salty). In large-scale regression analysis on over 900 words, this is exactly the pattern Connell and Lynott found. Lexical decision performance showed facilitation from visual strength alone (over and above lexical and sublexical variables), while word naming was facilitated by both visual and auditory strength. Moreover, these effects appeared independently of age of acquisition, after correction for multicollinearity ${ }^{4}$.

${ }^{4}$ While age of acquisition is sometimes presented as a lexico-semantic spoiler for pure semantic effects (e.g., Cortese \& Khanna, 2007; Monaghan \& Ellis, 2002), the opposite may instead be true, where a stronger perceptual basis to a word's meaning leads to earlier acquisition. Such an idea would be consistent with findings from Yu and Smith (2012) that the 
Perceptual strength measures have produced one other finding of note. While many semantic variables, including imageability (Yap, et al., 2012) and sensory experience ratings (Juhasz et al., 2011), produce larger effects in lexical decision than naming, perceptual strength does not. Rather, Connell and Lynott (2014a) found that the effect sizes for the two tasks were of comparable size. Because both tasks can benefit from a facilitatory boost due to the allocation of perceptual attention to the visual or auditory modality, there is no a priori reason to expect large differences in the size of the modality-specific effects observed in each task. However, such predictions and observations can only be made at the level of modality-specific processing proposed by the AMMA account, rather than at the level of undifferentiated sensorimotor simulation captured by measures such as imageability or sensory experience.

While there is evidence that perceptual strength outperforms other embodied semantic measures (imageability, sensory experience) in predicting visual word recognition behaviour, it is unclear how perceptual strength effects fit with action-based effects such as those captured by

names of objects that dominate the visual field are learned more quickly than names of less visually dominant objects, and - for the visual modality, at least - Connell and Lynott's data are supportive. In their study, age of acquisition was moderately correlated with visual strength $(r=$ $-.296)$ but very weakly with auditory strength $(r=-.041)$. This pattern of effects suggests that perceptual experience of an object is related to the age at which a word or concept is acquired, but the relationship is not uniform across perceptual modalities. Rather, children tend to learn labels for strongly visual concepts early on, and shift increasingly to weakly visual concepts as they get older, whereas the labels for strongly auditory concepts are learned at a relatively constant rate. However, more research is needed to determine how word learning varies with age in how it relies on other modalities of perceptual experience, such as touch, taste, and smell. 
body-object recognition and relative embodiment. The AMMA account currently concentrates on perception, but it may be possible for it to be extended to include a motor attention component, whereby the perceptual / motor attention implicitly involved in different reading tasks preactivates modality-specific cortices, leading to faster simulation of modality-specific information, which in turn facilitates recognition of a word. However, more research is certainly needed to test how perceptual and motor attention affect visual word recognition, and how modality-specific perceptual simulation might differentially interact with motor simulation in semantic content.

\section{Embodied Semantic Effects: Conclusions}

Having reviewed the evidence for a number of contrasting views of embodied meaning, it is possible to establish something of a pecking order among these perspectives in their ability to account for all of the existing embodied semantic effects on visual word recognition.

It seems clear that the weakest of the approaches discussed in accounting for the empirical data on visual word recognition concerns imagistic representations. Imageability effects themselves are explained, of course, as facilitation from the directness of connection between a word's verbal code and imagistic code: a more direct connection leads to more semantic activation. One could argue that body-object interaction, relative embodiment, and sensory experience effects could be accommodated in a dual coding account of imagistic representations, perhaps by perceptual and motor experience increasing the directness of connection between a particular verbal and imagistic code. However, one would then expect the increased directness of connections to be reflected in imageability ratings, yet body-object interaction, relative embodiment, and sensory experience effects are largely independent of imageability. A bigger problem for imagistic representations is posed by perceptual strength 
effects. Imageability effects are subsumed by perceptual strength, and dual-coding theory cannot explain why modality-specific perceptual strength effects vary by task (nor is it clear how it could be adapted to do so). If semantic content comprised imagistic representations as outlined by dual coding theory, where imagistic codes are functionally distinct from sensory systems during reading (e.g., Sadoski et al., 2012), then it could not accommodate selective facilitation effects such as the ability of auditory perceptual strength to facilitate word naming but not lexical decision.

The basic sensorimotor simulation approach to semantic content is more successful than imagistic representations in explaining the range of embodied semantic effects in visual word recognition. The extent of sensorimotor experience in a referent concept facilitates visual word recognition, where more is better: greater experience (as indexed by body-object interaction, relative embodiment, and sensory experience ratings) gives rise to greater semantic activation, and greater facilitation of word recognition. In terms of imageability effects, it is plausible that the effort of accessing this sensorimotor experience also plays a role: that is, the ease of sensorimotor simulation (as indexed by imageability ratings) may facilitate semantic activation as well as the degree of sensorimotor information comprising semantic content. As with imagistic representations, however, a general sensorimotor simulation approach to semantic content cannot explain modality-specific perceptual strength effects. Even with united perceptual and conceptual systems, a "more is better" perspective on sensorimotor simulation is too coarsegrained to explain why activation of semantic content varies according to interactions with modality-specific task demands.

Based on the evidence to date, embodied semantic effects are currently best explained by considering semantic content as sensorimotor simulation modulated by perceptual attention. The attentional modulation of meaning activation (AMMA) theory explains the task-specific, 
modality-specific perceptual strength effects that were problematic for the other accounts of semantic content: perceptual attention pre-activates modality-specific cortices, which leads to faster simulation of information in those modalities during semantic activation. Hence, it is the extent of sensorimotor experience in a referent concept, whose access is modulated by modalityspecific perceptual attention, that facilitates visual word recognition. In this way, AMMA also explains body-object interaction, relative embodiment, and sensory experience effects, although it is a matter for future research whether motor simulation is modulated by selective attention in like manner to perceptual simulation. Finally, since imageability effects are subsumed by perceptual strength effects in visual word recognition, the AMMA account does not need to make any additional assumptions to accommodate them.

\section{Issues for Models of Visual Word Recognition}

The embodied semantic effects reviewed in this chapter all show that visual word recognition is facilitated by the degree to which a word's semantic content involves perceptual or bodily experience. While many of these effects are broadly compatible with existing models of visual word recognition that allow early, low-level effects of semantic information, some particularly the modality-specific effects from attentional modulation - are not easily accommodated by current accounts.

Two of the most influential models of visual word recognition are the triangle model family (e.g., Harm \& Seidenberg, 2004; Plaut et al., 1996; Woollams, Lambon Ralph, Plaut, \& Patterson, 2007; based on the PDP model of Seidenberg \& McClelland, 1989) and the dual route cascaded model (DRC: Coltheart et al., 2001; Coltheart, Tree, \& Saunders, 2010). Both accounts agree with the general process of semantic effects earlier outlined (i.e., that feedback from semantic content facilitates processing orthographic and/or phonological representations of the 
word), but differ greatly on processing specifics and the necessity of semantic feedback.

The triangle model handles visual word recognition as a distributed process, where written words produce a pattern of activation across orthographic, phonological, and semantic components. A word is recognised based on the distributed pattern across all three components, though words with irregular spellings or pronunciations rely more on feedback from the semantic component to aid identification. The influence of semantic content on visual word recognition is therefore obligatory, at least to some extent, because the semantic component forms one corner of the triangle of distributed representations. In contrast, the DRC model splits visual recognition of known words into two processes: a direct route that runs directly from orthographic to phonological representation without semantic input, and an indirect route where the orthographic to phonological mapping runs via the semantic component (a separate, parallel route handles orthographic to phonological translation of unknown words). A word is recognised based on its presence in the orthographic and phonological lexicons, for which semantic feedback is an optional aid. Thus, in the DRC model, semantic content can influence visual word recognition, but is not necessary.

However, it should be noted that almost all testing and adjudication of these models have operated by implementing the orthographic and phonological components alone, with infrequent and incomplete inclusion of information in the semantic component. As such, both triangle and DRC models remain somewhat underspecified when it comes to explaining why semantic effects of any sort emerge. The AMMA account, which explains embodied semantic effects in terms of sensorimotor simulation modulated by perceptual attention, raises theoretical issues for these models that are incompatible with their current accounts of visual word recognition. 


\section{The Curse of Black Box Semantics}

Both the triangle and DRC models have tended to treat semantics as a black box; that is, a component considered entirely in terms of its inputs and outputs, with scant regard for the internal composition and workings. Since neither model has implemented a full semantics component $^{5}$, instead retaining it as a theoretical construct that can feed activation to orthographic and phonological representations, semantic information itself remains nebulous.

The main problem with black box semantics is that semantic effects are implicitly assumed to be uniform: any type of semantic information has the same effect. Regardless of what the semantic information might be - whether it relates to specific qualities of semantic content (Level 1), enumeration of content (Level 2), or word neighbourhood (Level 3) - it lights up the semantics box and feeds activation back to the orthographic/phonological representations. More semantic information leads to more feedback and more facilitation; an idea explicitly espoused by the perspective that semantic richness is what facilitates visual word recognition (Grondin et al., 2009; Pexman et al., 2002, 2008; Yap et al., 2011).

Evidence from Connell and Lynott (2014), showing that implicit perceptual attention

${ }^{5}$ Some triangle models have partly fleshed out a semantics component. Harm and Seidenberg (2004) implemented an effectively amodal form of semantic content by representing concepts as collections of features derived from Wordnet (e.g., dog had features such as mammal, has-part-tail). Dilkina et al. (2008) included separate visual and motor layers, where concepts' visual and action information was abstracted by constructing bit patterns to act as category prototypes (e.g., for mammals, fruit, furniture) and then probabilistically sampling around them to create category members. In neither of these models did the semantic component capture the extent of sensorimotor experience in a word's semantic content, and hence neither can model the sort of Level 1 embodied semantic effects discussed in this chapter. 
interacts with modality-specific aspects of meaning in facilitating word recognition, now strongly suggests that semantic activation cannot be bounded in this way. Rather, since the same perceptual systems that are focusing attention on decoding the orthographic/phonological information of the word are simultaneously simulating the meaning of the word, the consequence is that the discrete boundaries of the semantic component are forced to dissolve. For example, different modalities of perceptual strength in the referent concept have different semantic effects on lexical decision: visual strength facilitates while auditory strength does not. Auditory strength cannot be disregarded, however, as it does facilitate word naming. Visual and auditory information, at the very least, must therefore be distinguished in the semantic component.

Moreover, it is not possible for a model to capture modality-specific perceptual strength effects on visual word recognition if semantic representations remain separate from the visual or auditory input of a word form. According to the AMMA theory, it is the perceptual demands of the reading task - visual attention in lexical decision; visual and auditory attention in word naming - that determine whether visual or auditory semantic information facilitates recognition of the word. Perceptual systems must therefore be implemented in models of word reading, connected to or merged with the semantic component, in order to allow differential activation of modality-specific semantic information according to which modalities are engaged in the particular reading task.

In other words, there is no longer room for a cleanly bounded, undifferentiated semantics box in models of visual word recognition, which necessitate new and different structures to explain the modality-specific range of embodied semantic effects.

\section{Cold-Start Versus Warm-Start Semantic Access}

The triangle and DRC models share a common assumption that semantic activation 
operates from a cold start. That is, access to the meaning of a word does not begin until after the word has been presented because the semantic component relies on activation spreading from the orthographic / phonological components. In the special case of semantic priming, some aspects of word meaning can be already active when the preceding word was related. However, this activation is effectively left over from a previous trial, and is only useful to semantic access on the current trial when the semantic content of prime and target words overlap, such as by sharing features (e.g., Becker et al., 1997) or category membership (e.g., Collins \& Quillian, 1969). On most trials of single word presentation, outside the semantic priming paradigm, there is no such useful overlap of representations, and semantic activation starts "cold" after presentation of the word.

However, Connell and Lynott's (2014) findings mean that semantic activation operates from a warm start: some of the meaning of a word is always pre-activated by the implicit perceptual attention involved in visual word recognition. In the AMMA theory, semantic access depends on both the strength of modality-specific perceptual experience in the referent concept and the modality-specific perceptual attention implicitly engaged by the reading task. That is, the nature of expecting to process a written word engages visual attention, which pre-activates the visual system in anticipation of visual stimulus. Likewise, expecting to pronounce a word aloud engages auditory attention, which pre-activates the auditory system. Since the same modality-specific sensory system handles both perception and simulation of semantic content in that modality, lexical decision leads to pre-activation of visual semantic information, and word naming leads to pre-activation of both visual and auditory semantic information. Such semantic activation occurs regardless of semantic priming, and is in place before a the first trial even appears, resulting in semantic access starting "warm" when the word is presented.

It should be noted that these effects are not confined to concrete concepts with an obvious 
visual or auditory basis. Even very traditionally abstract concepts, such as zero, cause, or republic, still have visual and/or auditory presence (Connell \& Lynott, 2012; see also Ghio, Vaghi, \& Tettamanti, 2013, for evidence that motor experience from different effectors may also be relevant to abstract concepts). For example, zero scores 2.75 out of 5 on visual strength, indicating that people experience it by seeing, to a moderate extent (Lynott \& Connell, 2013). Indeed, there may be no such thing as truly aperceptual concepts. If so, then all words can benefit to some extent from warm-start semantic activation, even if the visually- and auditorilystrongest (depending on task) benefit the most. Current models of visual word recognition appear to have assumed cold-start semantic access as a default position, perhaps in the former absence of evidence to the contrary, and hence need to be adapted to accommodate warm-start activation. If models enfolded the perceptual system to account for modality-specific effects, as previously outlined in the discussion of black box semantics, then they would effectively implement warm-start semantic access.

\section{Task Distinctions}

Both the triangle and DRC models consider lexical decision as largely similar to word naming. In simplified terms, the orthographics of the word are first activated, then activation spreads to the semantic and phonological components, and feedback and feedforward connections allow activation to spread continually between orthography, phonology, and semantics. Lexical decision of "yes" is then triggered by orthographic activation (clearing a threshold in the triangle model, or finding a match in the DRC model), while word naming outputs correct pronunciation based on phonological activation (again, clearing a threshold in the triangle model, or finding a match in the DRC model). As such, there is no a priori distinction between tasks in how semantic activation emerges, and both tasks activate the same semantic 
representation for a particular word.

But from the perspective of perceptual attention, lexical decision and word naming are fundamentally different processes. As outlined by AMMA, different reading tasks require implicit perceptual attention on different modalities, which in turn affects (a) the semantic content that is activated in each task for a particular word, with preferential simulation in the currently-attended modalities, and (b) the degree of facilitation observed in each task for a particular word, according to the perceptual strength of its referent concept in the currentlyattended modalities. Take a word like noisy, which is strongly auditory (4.95 out of 5) but weak on other modalities (all less than 1.7 out of 5: Lynott \& Connell, 2009). In a lexical decision task, implicit perceptual attention will lead to warm-start simulation of visual information (see previous section), which will be of small benefit to noisy since so little of its semantic content is visual. In a word naming task, however, implicit perceptual attention will lead to warm-start simulation of auditory as well as visual information, which this time will benefit noisy quite a bit because auditory information makes up an important part of its semantic content. Hence, the task itself - along with modality-specific perceptual strength in semantic content - modulates semantic effects in visual word recognition; a pattern which current models cannot accommodate.

Moreover, both models prefer the direct orthographic $\rightarrow$ phonological path in word naming over the indirect orthographic $\rightarrow$ semantic $\rightarrow$ phonological path, particularly for frequent words with regular pronunciations. Accordingly, when it comes to naming, both models consider semantic effects to be somewhat optional for healthy participants (though they disagree as to the extent of such optionality). This position fits with previous observations that word naming seemed less susceptible to semantic effects than lexical decision (e.g., de Groot, 1989; Strain et al., 1995), and with the idea that lexical decision was more prone to semantic effects because 
semantic information can be used as a proxy for the word/nonword decision (Chumbley and Balota, 1984; see also Balota et al., 2004). Current findings, however, indicate otherwise. Connell and Lynott (2014) found that modality-specific perceptual strength effects were equally strong in lexical decision and naming tasks. Indeed, as indicated above with the noisy example, a particular word may sometimes have greater semantic facilitation in naming than in lexical decision. Modality-specific perceptual strength effects are therefore commonplace in word naming as well as in lexical decision, and not just for words with particularly tricky mappings from spelling to sound, which means that the indirect orthographic $\rightarrow$ semantic $\rightarrow$ phonological path may contribute more than is typically allowed in current models of visual word recognition.

In summary, word naming and lexical decision are different processes with different task goals, different implicit perceptual attention, and different prioritization of semantic information during meaning activation. Since the type of reading task itself modulates the profile of semantic information that is activated for a particular word by directing perceptual attention to specific modalities, the basic processes of lexical decision and word naming are not identical in their early stages in how they activate the semantic component. As outlined in the previous sections, models of visual word recognition would need to implement a modality-specific semantic system that was connected to or merged with a modality-specific perceptual system if they were to accommodate the pattern of task-specific embodied semantic effects. Furthermore, much recent theory adjudication between the triangle and DRC models has focused on the role of direct versus indirect paths in the degradation of word naming performance in semantic dementia (e.g., Coltheart et al., 2010; Woollams et al., 2007). We suggest that capturing semantic effects in typical adult reading, particularly the recent batch of modality-specific semantic effects, should be a necessary step before attempting to model impairment: one must learn to walk before trying to run. 


\section{Future Directions}

Recent years have seen a large increase in the range of embodied semantic effects in visual word recognition, which emerge from the perceptual and motor experiential basis of the referent concept. These effects have necessitated a more complex view of semantics than has been hitherto found in visual word recognition research, but can be explained by the AMMA theory's view of semantic content as a sensorimotor simulation modulated by perceptual attention. Moreover, the pervasiveness of semantic effects in normal word recognition is demonstrated by their robust appearance in megastudy regressions across hundreds of words. Word meaning matters to typical reading in typical circumstances, and is not just restricted to atypical classes of words with irregular spellings / pronunciations, or atypical groups of people with dyslexia or semantic dementia.

All the same, much work remains to be done. Larger studies must be conducted with the statistical power to disentangle modality-specific perceptual and motor effects, and to systematically determine their independence from other key variables such as age of acquisition (e.g., Connell \& Lynott, 2014a), as well as less-studied aspects of word learning such as mode of acquisition (e.g., whether meaning acquired linguistically versus perceptually: Wauters et al., 2003). More specific analysis of the attentional demands of different visual word recognition paradigms is needed to map the relationship between reading and semantic activation, since the extent of any facilitation from perceptual attention will depend on the exact task demands, available cognitive resources, and processing goals (Connell \& Lynott, 2012b, 2014b). Finally, research must address how emotion fits with the sensorimotor effects reviewed in this chapter, in order to determine whether valence effects on visual word recognition (e.g., Kuperman, Estes, Brysbaert, \& Warringer, 2014; Kousta, Vinson, \& Vigliocco, 2009) emerge from semantic feedback (i.e., positive and negative affective simulations feed more activation back to the 
orthographic/phonological representations of the word than do neutral affective simulations) or from automatic vigilance for negative stimuli (i.e., negative words engage attention for longer than positive words and delay making the lexical decision or naming response). As our understanding of the nature of conceptual representations grows, so too should our ability to capture how the meaning of words influences their processing. 


\section{References}

Balota, D. A., \& Chumbley, J. I. (1984). Are lexical decisions a good measure of lexical access? The role of word frequency in the neglected decision stage. Journal of Experimental Psychology: Human Perception and Performance, 10(3), 340.

Balota, D. A., Cortese, M. J., Sergent-Marshall, S. D., Spieler, D. H., \& Yap, M. J. (2004). Visual word recognition of single-syllable words. Journal of Experimental Psychology: General, 133, 283-316.

Barsalou, L. W. (1999). Perceptual symbol systems. Behavioral and Brain Sciences, 22, 577660.

Barsalou, L. W. (2009). Simulation, situated conceptualization, and prediction. Philosophical Transactions of the Royal Society B: Biological Sciences, 364, 1281-1289.

Becker, S., Moscovitch, M., Behrmann, M., \& Joordens, S. (1997). Long-term semantic priming: a computational account and empirical evidence. Journal of Experimental Psychology: Learning, Memory, and Cognition, 23(5), 1059.

Binder, J. R., Westbury, C. F., McKiernan, K. A., Possing, E. T., \& Medler, D. A. (2005). Distinct brain systems for processing concrete and abstract words. Journal of Cognitive Neuroscience, 17, 905-917.

Blazely, A. M., Coltheart, M., \& Casey, B. J. (2005). Semantic impairment with and without surface dyslexia: Implications for models of reading. Cognitive Neuropsychology, 22(6), $695-717$.

Borowsky, R., \& Masson, M. E. (1996). Semantic ambiguity effects in word identification. Journal of Experimental Psychology: Learning, Memory, and Cognition, 22(1), 63.

Brown, G. D., \& Watson, F. L. (1987). First in, first out: Word learning age and spoken word frequency as predictors of word familiarity and word naming latency. Memory \& 
Cognition, 15, 208-216.

Buchanan, L., Westbury, C., \& Burgess, C. (2001). Characterizing semantic space:

Neighborhood effects in word recognition. Psychonomic Bulletin \& Review, 8(3), 531544.

Chumbley, J. I., \& Balota, D. A. (1984). A word's meaning affects the decision in lexical decision. Memory \& Cognition, 12(6), 590-606.

Clark, J. M., \& Paivio, A. (2004). Extensions of the Paivio, Yuille, and Madigan (1968) norms. Behavior Research Methods, 36, 371-383.

Collins, A. M., \& Quillian, M. R. (1969). Retrieval time from semantic memory. Journal of Verbal Learning and Verbal Behavior, 8(2), 240-247.

Coltheart, M., Rastle, K., Perry, C., Langdon, R., Ziegler, J. (2001). DRC: A dual route cascaded model of visual word recognition and reading aloud. Psychological Review, 108, 204-256

Coltheart, M., Tree, J. J., \& Saunders, S. J. (2010). Computational modeling of reading in semantic dementia: comment on Woollams, Lambon Ralph, Plaut, and Patterson (2007). Psychological Review, 117, 256-272.

Connell, L., \& Lynott, D. (in prep.). Do we know what we're simulating? Information loss on introspective examination of perceptual experience. Manuscript in preparation.

Connell, L., and Lynott, D. (2012a). Strength of perceptual experience predicts word processing performance better than concreteness or imageability. Cognition, 125, 452-465.

Connell, L., \& Lynott, D. (2012b). When does perception facilitate or interfere with conceptual processing? The effect of attentional modulation. Frontiers in Psychology, 3(474), 1-3.

Connell, L., \& Lynott, D. (2014a). I see/hear what you mean: Semantic activation in visual word recognition depends on perceptual attention. Journal of Experimental Psychology: General, 143(2), 527. 
Connell, L., \& Lynott, D. (2014b). Principles of Representation: Why You Can't Represent the Same Concept Twice. Topics in Cognitive Science, 6, 390-406.

Connell, L., Lynott, D., \& Dreyer, F. (2012). A functional role for modality-specific perceptual systems in conceptual representations. PloS ONE, 7(3), e33321.

Cortese, M. J., \& Balota, D. A. (2012). Visual word recognition in skilled adult readers. In M. J. Spivey, K. McRae, \& M. F. Joanisse (Eds.), The Cambridge Handbook of Psycholinguistics (pp. 159-185). Cambridge, MA: Cambridge University Press.

Cortese, M. J., \& Khanna, M. M. (2008). Age of acquisition ratings for 3,000 monosyllabic words. Behavior Research Methods, 40, 791-794.

Cortese, M. J., \& Schock, J. (2013). Imageability and age of acquisition effects in disyllabic word recognition. Quarterly Journal of Experimental Psychology, 66, 946-972.

Coventry, K. R., Lynott, D., Cangelosi, A., Monrouxe, L., Joyce, D., \& Richardson, D. C. (2010). Spatial language, visual attention, and perceptual simulation. Brain and Language, 112(3), 202-213.

de Groot, A. M. B. (1989). Representational aspects of word imageability and word frequency as assessed through word association. Journal of Experimental Psychology: Learning, Memory, and Cognition, 15, 824-845

Dilkina, K., McClelland, J. L., \& Plaut, D. C. (2008). A single-system account of semantic and lexical deficits in five semantic dementia patients. Cognitive Neuropsychology, 25, 136164.

Duñabeitia, J. A., Avilés, A., \& Carreiras, M. (2008). NoA's ark: Influence of the number of associates in visual word recognition. Psychonomic Bulletin \& Review, 15(6), 1072-1077.

Ellis, A. W., \& Monaghan, J. (2002). Reply to Strain, Patterson, and Seidenberg (2002). Journal of Experimental Psychology: Learning, Memory, and Cognition, 28, 215-220. 
Fliessbach, K., Weis, S., Klaver, P., Elger, C. E., \& Weber, B. (2006). The effect of word concreteness on recognition memory. Neuroimage, 32, 1413-1421.

Gallese, V. (2009). Motor abstraction: A neuroscientific account of how action goals and intentions are mapped and understood. Psychological Research PRPF, 73, 486-498.

Ghio, M., Vaghi, M. M. S., \& Tettamanti, M. (2013). Fine-grained semantic categorization across the abstract and concrete domains. PLoS ONE, 8(6), e67090.

Grondin, R., Lupker, S. J., \& McRae, K. (2009). Shared features dominate semantic richness effects for concrete concepts. Journal of Memory and Language, 60(1), 1-19.

Hargreaves, I. S., \& Pexman, P. M. (2014). Get rich quick: The signal to respond procedure reveals the time course of semantic richness effects during visual word recognition. Cognition, 131(2), 216-242.

Harm, M. W., \& Seidenberg, M. S. (2004). Computing the meanings of words in reading: cooperative division of labor between visual and phonological processes. Psychological Review, 111(3), 662.

Hino, Y., \& Lupker, S. J. (1996). Effects of polysemy in lexical decision and naming: An alternative to lexical access accounts. Journal of Experimental Psychology: Human Perception and Performance, 22(6), 1331.

James, C. T. (1975). The role of semantic information in lexical decisions. Journal of Experimental Psychology: Human Perception and Performance, 1, 130-136.

Juhasz, B. J., \& Yap, M. J. (2013). Sensory experience ratings for over 5,000 mono-and disyllabic words. Behavior Research Methods, 45(1), 160-168.

Juhasz, B. J., Yap, M. J., Dicke, J., Taylor, S. C., \& Gullick, M. M. (2011). Tangible words are recognized faster: The grounding of meaning in sensory and perceptual systems. The Quarterly Journal of Experimental Psychology, 64(9), 1683-1691. 
Keuleers, E., Lacey, P., Rastle, K., \& Brysbaert, M. (2012). The British Lexicon Project: Lexical decision data for 28,730 monosyllabic and disyllabic English words. Behavior Research Methods, 44(1), 287-304.

Kousta, S. T., Vinson, D. P., \& Vigliocco, G. (2009). Emotion words, regardless of polarity, have a processing advantage over neutral words. Cognition, 112, 473-481.

Kuperman, V., Estes, Z., Brysbaert, M., \& Warriner, A. B. (2014). Emotion and language:

Valence and arousal affect word recognition. Journal of Experimental Psychology: General, 143, 1065-1081.

Lynott, D., \& Connell, L. (2009). Modality exclusivity norms for 423 object properties. Behavior Research Methods, 41, 558-564.

Lynott, D., \& Connell, L. (2013). Modality exclusivity norms for 400 nouns: The relationship between perceptual experience and surface word form. Behavior Research Methods, 45, 516-526.

Meteyard, L., Rodriguez Cuadrado, S., Bahrami, B., \& Vigliocco, G. (2012). Coming of age: A review of embodiment and the neuroscience of semantics. Cortex, 48, 788-804.

Mirman, D., \& Magnuson, J. S. (2008). Attractor dynamics and semantic neighborhood density: processing is slowed by near neighbors and speeded by distant neighbors. Journal of Experimental Psychology: Learning, Memory, and Cognition, 34(1), 65.

Monaghan, J., \& Ellis, A. W. (2002). What exactly interacts with spelling-sound consistency in word naming?. Journal of Experimental Psychology: Learning, Memory, and Cognition, $28(1), 183$.

Paivio, A. (1986). Mental representations: A dual coding approach. Oxford, UK: Oxford University Press. 
Paivio, A. (2007). Mind and its evolution: A dual coding theoretical approach. Mahwah, NJ: Erlbaum.

Paivio, A., Yuille, J. C., \& Madigan, S. A. (1968). Concreteness, imagery, and meaningfulness values for 925 nouns. Journal of Experimental Psychology,76(1p2), 1.

Pexman, P. M., Lupker, S. J., \& Hino, Y. (2002). The impact of feedback semantics in visual word recognition: Number-of-features effects in lexical decision and naming tasks. Psychonomic Bulletin \& Review, 9(3), 542-549.

Pexman, P. M., Hargreaves, I. S., Siakaluk, P. D., Bodner, G. F., \& Pope, J. (2008). There are many ways to be rich: Effects of three measures of semantic richness on visual word recognition. Psychonomic Bulletin \& Review, 15, 161-167.

Plaut, D. C., McClelland, J. L., Seidenberg, M. S., \& Patterson, K. (1996). Understanding normal and impaired word reading: Computational principles in quasi-regular domains. Psychological Review, 103, 56-115.

Pulvermüller, F. (2005). Brain mechanisms linking language and action. Nature Reviews Neuroscience, 6, 576-582.

Rodd, J. M., Gaskell, M. G., \& Marslen-Wilson, W. D. (2004). Modelling the effects of semantic ambiguity in word recognition. Cognitive Science, 28, 89-104.

Rogers, T. T., Lambon Ralph, M. A., Hodges, J. R., \& Patterson, K. (2004). Natural selection: The impact of semantic impairment on lexical and object decision. Cognitive Neuropsychology, 21(2-4), 331-352.

Sabsevitz, D. S., Medler, D. A., Seidenberg, M., \& Binder, J. R. (2005). Modulation of the semantic system by word imageability. Neuroimage, 27, 188-200.

Sadoski, M., McTigue, E. M., \& Paivio, A. (2012). A dual coding theoretical model of decoding in reading: subsuming the LaBerge and Samuels model. Reading Psychology, 33(5), $465-$ 
496.

Seidenberg, M. S., \& McClelland, J. L. (1989). A distributed, developmental model of word recognition and naming. Psychological Review, 96(4), 523.

Siakaluk, P. D., Pexman, P. M., Aguilera, L., Owen, W. J., \& Sears, C. R. (2008). Evidence for the activation of sensorimotor information during visual word recognition: The bodyobject interaction effect. Cognition, 106(1), 433-443.

Sidhu, D. M., Kwan, R., Pexman, P. M., \& Siakaluk, P. D. (2014). Effects of relative embodiment in lexical and semantic processing of verbs. Acta psychologica, 149, 32-39.

Solomon, K. O., \& Barsalou, L. W. (2004). Perceptual simulation in property verification. Memory \& Cognition, 32(2), 244-259.

Strain, E., Patterson, K., \& Seidenberg, M. S. (1995). Semantic effects in single-word naming. Journal of Experimental Psychology: Learning, Memory, and Cognition, 21(5), 1140.

Strain, E., Patterson, K., \& Seidenberg, M. S. (2002). Theories of word naming interact with spelling-sound consistency. Journal of Experimental Psychology: Learning, Memory, and Cognition, 28, 207-214.

Tillotson, S. M., Siakaluk, P. D., \& Pexman, P. M. (2008). Body_object interaction ratings for 1,618 monosyllabic nouns. Behavior Research Methods,40(4), 1075-1078.

Tousignant, C., \& Pexman, P. M. (2012). Flexible recruitment of semantic richness: context modulates body-object interaction effects in lexical-semantic processing. Frontiers in Human Neuroscience, 6: 53.

Wauters, L.N., Tellings, A.E.J.M., van Bon, W.H.J., \& van Haaften, A.W. (2003). Mode of acquisition of word meanings: The viability of a theoretical construct. Applied Psycholinguistics, 24, 385-406.

Wellsby, M., \& Pexman, P. M. (2014). The influence of bodily experience on children's 
language processing. Topics in Cognitive Science, 6(3), 425-441.

Wellsby, M., Siakaluk, P. D., Owen, W. J., \& Pexman, P. M. (2011). Embodied semantic processing: the body-object interaction effect in a non-manual task. Language and Cognition, 3(1), 1-14.

Woollams, A. M. (2005). Imageability and ambiguity effects in speeded naming: convergence and divergence. Journal of Experimental Psychology: Learning, Memory, and Cognition, $31(5), 878$.

Woollams, A. M., Ralph, M. A. L., Plaut, D. C., \& Patterson, K. (2007). SD-squared: on the association between semantic dementia and surface dyslexia. Psychological Review, $114(2), 316$.

Yap, M. J., Tan, S. E., Pexman, P. M., \& Hargreaves, I. S. (2011). Is more always better? Effects of semantic richness on lexical decision, speeded pronunciation, and semantic classification. Psychonomic Bulletin \& Review, 18(4), 742-750.

Yap, M. J., Pexman, P. M., Wellsby, M., Hargreaves, I. S., \& Huff, M. J. (2012). An abundance of riches: cross-task comparisons of semantic richness effects in visual word recognition. Frontiers in Human Neuroscience, 6(72), 1-10.

Yu, C., \& Smith, L. B. (2012). Embodied attention and word learning by toddlers. Cognition, $125,244-262$. 